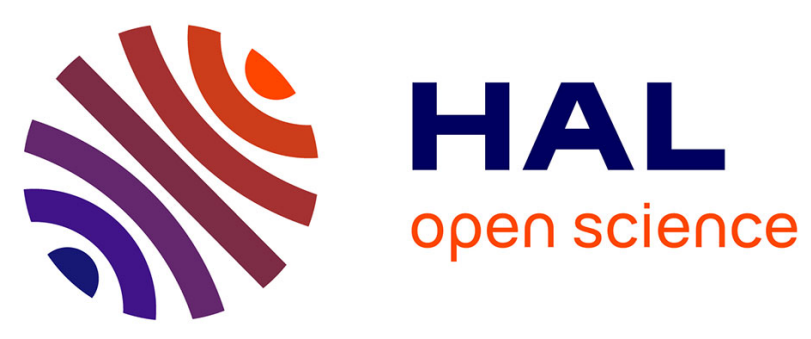

\title{
Two-Discipline Optimization
}

Jean-Antoine Desideri

\section{To cite this version:}

Jean-Antoine Desideri. Two-Discipline Optimization. Piotr Breitkopf and Rajan Filomeno Coelho. Multidisciplinary Design Optimization in Computational Mechanics, ISTE and John Wiley, pp.287320, 2010, 9781848211384. inria-00537115

\section{HAL Id: inria-00537115 https://hal.inria.fr/inria-00537115}

Submitted on 17 Nov 2010

HAL is a multi-disciplinary open access archive for the deposit and dissemination of scientific research documents, whether they are published or not. The documents may come from teaching and research institutions in France or abroad, or from public or private research centers.
L'archive ouverte pluridisciplinaire HAL, est destinée au dépôt et à la diffusion de documents scientifiques de niveau recherche, publiés ou non, émanant des établissements d'enseignement et de recherche français ou étrangers, des laboratoires publics ou privés. 


\section{Multidisciplinary Design Optimization in Computational Mechanics}

OMD Partners

April 29, 2009 


\section{Two-Discipline Optimization}

A methodology for the numerical treatment of a two-objective minimization problem, possibly subject to equality constraints is proposed. Such a problem is a basic step in multidisciplinary optimization, sometimes referred to as concurrent engineering. Here, we consider the case where one criterion to be minimized, $J_{A}$, is preponderant over the second, $J_{B}$, that is, either more critical or more fragile. The problem is formulated as a parametric optimization in which the two criteria are smooth functions of a common design vector $Y \in \mathbb{R}^{N}$. The numerical procedure is made in two steps.

In the first step, the criterion associated with the preponderant criterion $J_{A}(Y)$, or primary discipline, is minimized first, alone, to full convergence by hypothesis, yielding the design vector $Y_{A}^{*}$. As a result, the gradient vector, $\nabla J_{A}^{*}$, the Hessian matrix, $H_{A}^{*}$, and the $K$ constraint gradients, $\nabla g_{k}^{*}$ are assumed to be known at $Y=Y_{A}^{*}$. In practice, such information may be difficult to calculate exactly when the finitedimensional parametric formulation is the result of discretizing functionals of the distributed solution of a complex set of partial differential equations, as it is the case in the prototype example of aerodynamic optimum-shape design; then, possibly, but not necessarily, the exact derivatives can be replaced by approximations through metamodeling of the functionals.

In preparation of the second step, the entire parametric space is then split into two supplementary subspaces on the basis of the analysis of the second variation of the primary functional. The construction is such that infinitesimal perturbations in the design vector $Y$ about $Y_{A}^{*}$ lying in the second subspace, whose dimension $p$ is adjustable ( $p \leq N-K$ ), cause potentially the least degradation of the primary functional value. In other words, the second subspace is the subspace of dimension $p$ of least sensitivity of the preponderant criterion $J_{A}$.

Chapter written by Jean-Antoine DÉSIDÉRI . 
In the second step of the optimization, a Nash equilibrium [NAS 51] is sought between the two disciplines by introducing two virtual players, each one in charge of minimizing its own criterion, $J_{A}$ or $J_{B}$, w.r.t. a small set of parameters that generates one of the above two supplementary subspaces. In this way, the secondary criterion is potentially reduced, while not increasing unduly the primary criterion from its initial minimum.

The Nash game is given a particular form in which a continuation parameter $\varepsilon$ $(0 \leq \varepsilon \leq 1)$ is introduced. The optimum solution $Y_{A}^{*}$ achieved at completion of the first step of the optimization is proved to be a Nash equilibrium solution of our formulation for $\varepsilon=0$. Thus, as $\varepsilon$ increases from 0 to 1 , the formulation provides a continuum of Nash equilibrium solutions, corresponding to a smooth introduction of the trade-off between the two disciplines. Along the continuum, the initial derivative of the primary functional w.r.t. $\varepsilon$ is also proved to be equal to zero, which can be viewed as a robust design result. In practice this offers the designer the possibility to elect a design point along the continuum.

Lastly we observe that the hierarchy introduced above between the criteria is applied to the split of territory in preparation of a Nash game, which is by essence symmetrical. The bias is therefore different in nature from the unsymmetrical treatment of the variables introduced in a Stackelberg-type game [BAS 95].

Our formulation was first demonstrated in the simple case of the minimization of two quadratic forms in $\mathbb{R}^{4}$ subject to a linear or a nonlinear equality constraint in [DÉS 07a]. The methodology is illustrated here by the treatment a difficult exercise of a generic aircraft wing shape optimization w.r.t. two criteria, one representative of the aerodynamic performance (drag) and the other of the structural design (average stress) taken from B. Abou El Majd's doctoral thesis [Abo 07].

\subsection{Pareto optimality, game strategies and split of territory in multiobjective optimization}

In the engineering office, the optimization problems that are raised by designers of complex systems are by nature multiobjective. For instance, in aerodynamic shape optimization for the design of commercial airplanes, one focus is the maximization of lift in the critical phase of take-off or landing, another is drag in the cruise regime since it directly determines kerosene consumption or range, but other criteria are also important : those related to stability or maneuverability and linked to aerodynamic moments, or manufacturing criteria, etc. Evidently, the resulting multiobjective optimization problems are inevitably also multipoint, since they are associated with different flight regimes (different Mach numbers and angles of attack) and configurations (e.g. possible deployment of special high-lift devices). Consequently, the accurate evaluation of such criteria by means of high-fidelity models requires the 
efficient simulation of several flowfields by the numerical approximation of the gasdynamics equations, typically by finite volumes. Lastly, different couplings of aerodynamics with other physical phenomena are also crucial in the analysis of structural deformation, stress and fatigue, dynamic fluid-structure interaction, acoustics, thermal, etc. These aspects can be treated in various ways with advanced numerical procedures. For example, in her doctoral thesis[MAR 08], M. Marcelet, in preparation of an aerodynamic aircraft wing shape optimization, has considered a model in which the compressible turbulent Navier-Stokes equations have been used to compute the three-dimensional flow about the wing, whereas the structure has been modeled as a beam subject to bending and torsion under the aerodynamic forces, and thus established the expression for the discrete gradient of aerodynamic coefficients. In this area, where functional gradients of complex coupled discrete systems are calculated, Automatic Differentiation as it is more and more routinely developed in tools such as TAPENADE (cf. http://www-sop.inria.fr/tropics), is expected to become increasingly useful. Another form of multidisciplinary design consists in considering a multiobjective problem in which the various objectives share a common set of design variables, the so-called "public variables" of the DIVE approach of Chapter 7, such as those representing a shape parametrically when the shape influences more than one discipline. How should the public variables be optimized concurrently to account for antagonistic criteria originating from different disciplines? This chapter focuses on this question sometimes referred to as "concurrent engineering". In optimum-shape design, often the different physical phenomena are accurately modeled by partial-differential equations to be solved in domains that are identical or distinct but share a common geometrical boundary at which appropriate conditions are enforced and whose shape is to be optimized. Besides the case of the aerostructural design of an aircraft wing cited above, in the design of a stealth airplane, one would optimize the wing-shape w.r.t. an appropriate aerodynamic criterion, or several such criteria, concurrently with an electromagnetic criterion, such as cross-radar section (RCS) reduction. In the latter case, both distributed P.D.E. systems are formulated in the domain exterior to the aircraft, but have very different computational characteristics in particular concerning the mesh requirements.

In the area of pure numerical simulation of multidisciplinary coupled systems, the computational cost to evaluate a configuration may be very high. A fortiori, in multidisciplinary optimization, one is led to evaluate a number of different configurations to iterate on the design parameters. This observation motivates the search for the most innovative and computationally efficient approaches in all the sectors of the computational chain : at the level of the solvers (using a hierarchy of physical models), the meshes and geometrical parameterizations for shape, or shape deformation, the implementation (on a sequential or parallel architecture; grid computing), and the optimizers (deterministic or semi-stochastic, or hybrid; synchronous, or asynchronous).

Classically, the simplest way to account for several criteria simultaneously consists in agglomerating them all in a single performance index weighting each criterion with 
an appropriate coefficient, or weight. For example, with two criteria $J_{A}$ and $J_{B}$, consider :

$$
J=\alpha \frac{J_{A}}{J_{A}^{0}}+\beta \frac{J_{B}}{J_{B}^{0}}
$$

where $J_{A}^{0}$ and $J_{B}^{0}$ are reference values, for example, those associated with an initial design. Here, $\alpha$ and $\beta$ are positive weights to be chosen somehow. This approach is very commonly-used, particularly when an initial good design is provided and only a better or slightly different optimum is to be sought. However, the construction of the agglomerated criterion involves a large amount of arbitrariness, in particular (but not only) w.r.t. the weights $\alpha$ and $\beta$ that can strongly influence the result and require to be calibrated by an experienced practitioner. Thus, this approach is poorly general, physically or mathematically relevant.

An alternative to the unique criterion by agglomeration of several objective functions, consists of a two-step process in which each criterion is first optimized alone, possibly under constraints; for the above two-objective problem, one thus gets $J_{A}^{*}$ and $J_{B}^{*}$ as the solutions to two independent single-objective optimizations. Then, in the second step, one solves the following single-objective constrained problem :

$$
\min p
$$

subject to the following inequality constraints :

$$
J_{A} \leq J_{A}^{*}+\alpha p \quad \text { and } \quad J_{B} \leq J_{B}^{*}+\beta p
$$

In this alternative, assuming all the cited single-objective problems make sense separately, without physical coupling, the difficulty is here to treat a problem with functional inequality constraints of physically-different nature. Additionally, the same arbitrariness resides in the calibration of the weights $\alpha$ and $\beta$.

A real alternative to the unique agglomerated objective approach, is to establish the front of Pareto-optimal solutions. To introduce this, we first recall the notion of dominance and non-dominance :

Definition : When considering the minimization of several criteria concurrently $\left(J_{A}\right.$, $J_{B}$, etc), a design point $D^{(1)}$ in the parameter space is said to dominate the design $D^{(2)}$, which we denote as follows :

$$
D^{(1)} \succ D^{(2)}
$$

iff, for all the criteria $J$ to be minimized, the following holds :

$$
J\left[D^{(1)}\right] \leq J\left[D^{(2)}\right],
$$

and if, for at least one criterion, the inequality is strict. Inversely, if instead :

$$
D^{(1)} \nsucc D^{(2)} \text {, and } D^{(2)} \nsucc D^{(1)} \text {, }
$$


the two design-points $D^{(1)}$ and $D^{(2)}$ are said to be non-dominated.

This notion can be used to sort a collection, or population of design-points evaluated w.r.t. the various criteria $J_{A}, J_{B}$, etc, according to the so-called Pareto fronts. The first front is made of all the design-points dominated by no other; the second, the front of those dominated by no other in the remaining set; etc. The result of this sorting process is sketched at FIG. 1.

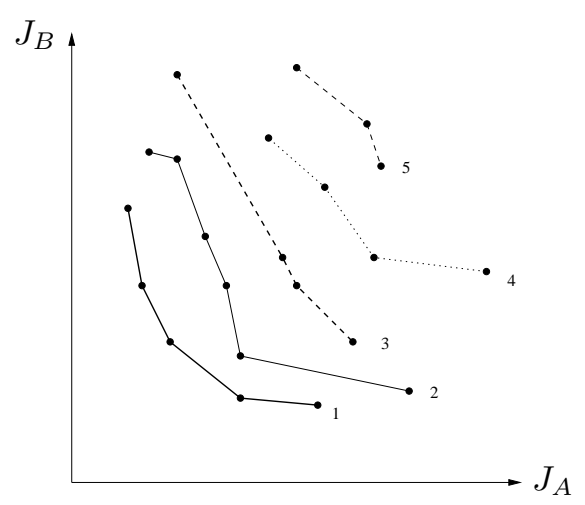

Figure 1. Sketch of a population of design-points sorted in Pareto fronts

Relying on this sorting process, Srinivas and Deb [SRI 95] have proposed the genetic algorithm NSGA (Non-dominated Sorting Genetic Algorithm) which utilizes essentially the front index as the fitness function, the engine of the GA. Goldberg [GOL 89] improved the method by introducing a niching technique in order to prevent the accumulation of non-dominated design-points on a given front. To illustrate the NSGA, we present an experiment made by Marco et al [MAR 99] in which an airfoil shape was optimized to reduce drag (in transonic flow conditions) and maximize lift (in subsonic flow conditions) concurrently. The NSGA was implemented in two independent experiments corresponding to finite-volume simulations of the compressible Euler equations using different meshes, a coarse and a fine. The totality of the design-points accumulated during the successive generations in the two experiments indistinctly, are represented on FIG. 2 a). In each experiment, the set of design-points does not cover the entire quarter plane : not all pairs $\left(J_{A}, J_{B}\right)$ can be achieved by the system. The boundary of the domain of realizable pairs is made of Pareto-optimal solutions. The corresponding two (discrete) fronts and the associated shapes (for the fine-mesh experiment only) are depicted on FIG. 2 b) and c).

For a more detailed and mathematical discussion on Pareto fronts and Pareto optimality, the reader is directed to the excellent K.M. Miettinen's textbook [MIE 99]. 
8 Multidisciplinary Design Optimization in Computational Mechanics

a) Realizable design-point accumulation by application of the NSGA (independent Eulerian flow simulations on a coarse and a fine meshes)

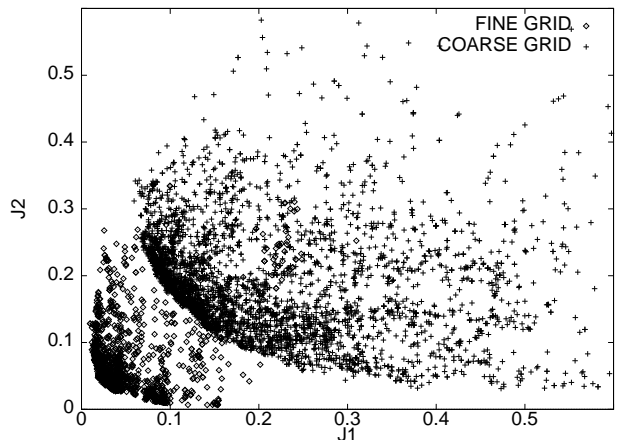

b) Discrete fronts of Pareto-optimal solutions (coarse and fine meshes)

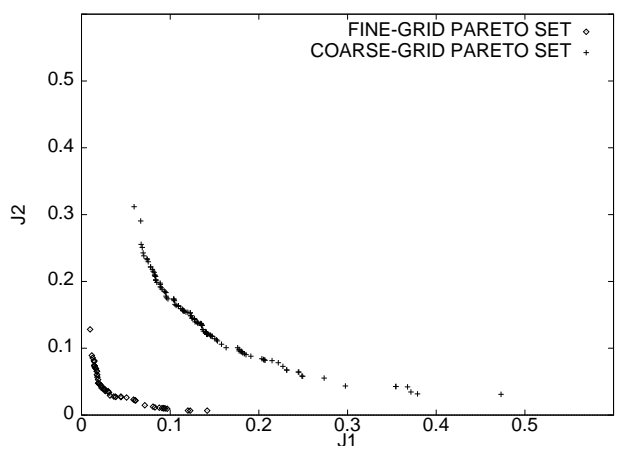

c) Shapes associated with the Pareto-optimal solutions (fine mesh)

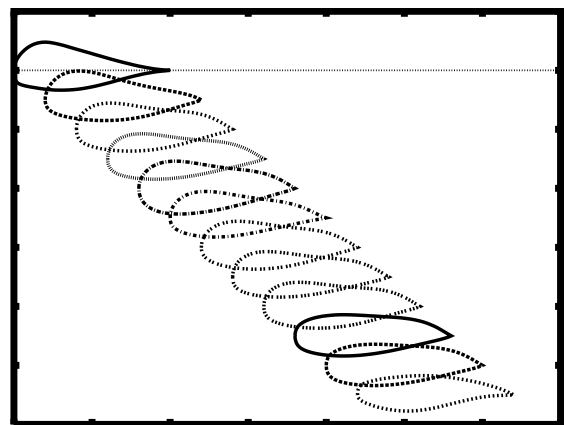

Figure 2. An illustration of the NSGA in which an airfoil shape is optimized to reduce drag and maximize lift concurrently 
This experiment allows us to point out the principal merits and weaknesses of this approach. The method provides the designer with a rich and unbiased information on the behavior of the criteria when the parameters vary, but one can also regret the lack of hierarchy between the Pareto-optimal solutions, among which a definite operating design-point requires to be elected on the basis of some other criterion still to be introduced. Other experiments in literature have shown that the method is very general since it has been applied to cases where the Pareto-equilibrium front was either non-convex or discontinuous. On the other hand, the computational cost of a standard application of the NSGA is fairly high since a large number of configurations ought to be evaluated, if an accurate identification of the front is sought. In our example, this was achieved by instantiations of a two-dimensional Eulerian flow code for purpose of demonstration; however today, realistic flow simulations about aircraft wings are based on three-dimensional turbulent Navier-Stokes equations. The cost-efficiency issue can be somewhat attenuated by the usage of parallel computing, which is possible at several levels : the parallelization of the analysis code by domain decomposition, the natural parallelization of its independent instantiations, as well as the parallelization the crossover operator in the GA [MAR 00].

When the front of Pareto-optimal solutions is convex and smooth, it may be possible to identify it pointwise, by treating all but one criterion as equality constraints, as depicted on FIG. 3. However this approach is much less general since, as mentioned before, functional constraints are difficult to implement; additionally, the identification is logically complex in cases of more than two objectives.

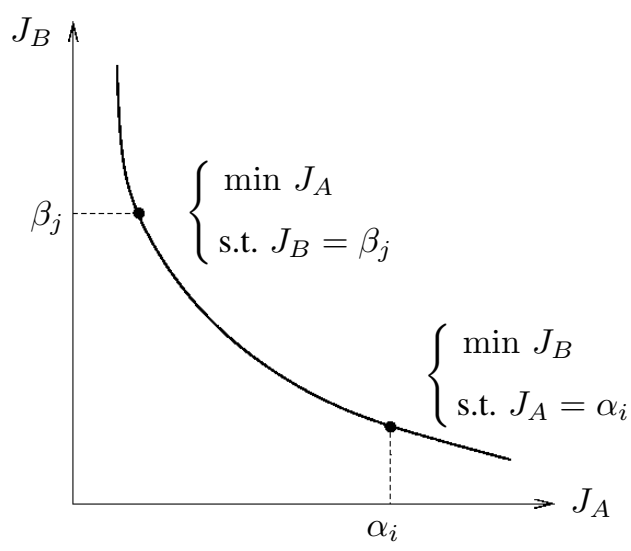

Figure 3. Schematic of a Pareto front pointwise identification by the treatment of certain criteria as equality constraints 
An alternate treatment of multiobjective problems that circumvents the usually very arbitrary question of adjusting penalty constants in the agglomerated-criterion approach, and that is much more economical than an NSGA-type method to establish the Pareto-equilibrium front, consists in simulating a dynamic game in which the design variables are split in complementary subsets and distributed to virtual players as individual strategies. Symmetrical as well as unsymmetrical (or hierarchical) games can be considered [NAS 51], [BAS 95]. In a symmetrical Nash game [NAS 51], each player accommodates its own strategy to the other players strategies to optimize only one criterion. If an equilibrium point is reached, a trade-off between the various criteria is achieved.

In his doctoral thesis, B. Abou El Majd [Abo 07] has realized a number of aerostructural shape-optimization exercises related to a generic business-jet wing using either Nash or Stackelberg games, some of which are reported here for illustration.

In this chapter, we focus on the symmetrical formulation of Nash games involving two players $A$ and $B$ controlling the subvectors $Y_{A}$ and $Y_{B}$ composing the complete vector of design variables :

$$
Y=\left(Y_{A}, Y_{B}\right)
$$

In this case, the vector $\bar{Y}=\left(\bar{Y}_{A}, \bar{Y}_{B}\right)$ is said to realize a Nash equilibrium of the criteria $J_{A}$ and $J_{B}$, iff :

$$
\bar{Y}_{A}=\operatorname{Argmin}_{Y_{A}} J_{A}\left(Y_{A}, \bar{Y}_{B}\right)
$$

and symmetrically :

$$
\bar{Y}_{B}=\operatorname{Argmin}_{Y_{B}} J_{B}\left(\bar{Y}_{A}, Y_{B}\right)
$$

This formulation is inspired by the negotiation mechanism of which economics and social sciences provide numerous examples.

The Nash equilibrium-point can be achieved by the following parallel algorithm [TAN 07] :

Step 1 : Initialize both subvectors :

$$
Y_{A}:=Y_{A}^{(0)} \quad Y_{B}:=Y_{B}^{(0)}
$$

Step 2: Perform in parallel optimization iterations of both subsystems (by independent and generally different analysis and optimization methods) : 
- Retrieve and maintain fixed

$$
Y_{B}=Y_{B}^{(0)}
$$

- Perform $K_{A}$ minimization steps of $J_{A}\left(Y_{A}, Y_{B}^{(0)}\right)$ by iterating on $Y_{A}$ alone and get $Y_{A}^{\left(K_{A}\right)}$.
- Retrieve and maintain fixed

$$
Y_{A}=Y_{A}^{(0)}
$$

- Perform $K_{B}$ minimization steps of $J_{B}\left(Y_{A}^{(0)}, Y_{B}\right)$ by iterating on $Y_{B}$ alone and get $Y_{B}^{\left(K_{B}\right)}$.

Step 3 : Update both subvectors in preparation of the information exchange :

$$
Y_{A}^{(0)}:=Y_{A}^{\left(K_{A}\right)} \quad Y_{B}^{(0)}:=Y_{B}^{\left(K_{B}\right)}
$$

and go back to Step 2 or stop (at equilibrium).

Note that in practice, under-relaxation is very often essential to convergence. This point is particularly critical when the two criteria $J_{A}$ and $J_{B}$ originate from different physical disciplines associated with different dependencies and scales, as it is the case for optimum design w.r.t. aerodynamics and structural mechanics, or electromagnetics. However, certain rather general mathematical stabilization techniques exist; see for example [ATT 07].

Important remark : assume that $\bar{Y}=\left(\bar{Y}_{A}, \bar{Y}_{B}\right)$ realizes a Nash equilibrium of the criteria $J_{A}$ and $J_{B}$, and let $\Phi$ and $\Psi$ be some arbitrary but smooth and strictlymonotone increasing functions; then, evidently, $\bar{Y}$ also realizes a Nash equilibrium of the criteria $\Phi\left[J_{A}\right]$ and $\Psi\left[J_{B}\right]$. In other words, the notion of Nash equilibrium is independent of the scales and the physical dimensions used to measure the criteria : for example, replacing $J$ by $J^{\alpha}$ or $\exp (J)$ has no effects other than, perhaps, a different conditioning of the numerical procedures. By this invariance property, the Nash game formulation contrasts outstandingly from the agglomerated criterion approach in which dimensioning the penalty constants has a strong, and usually unknown influence on the solution. The equilibrium solution, unique or not, is only determined by the split of the design vector, which is here referred to as the split of territory by which each virtual player is allocated a subspace of action, or territory.

Under the thrust of Jacques Périaux, this approach has been tested successfully over a number of cases related to optimum design in aeronautics, in particular within the framework of the Jacques-Louis Lions Laboratory common to the University of Paris 6 and Dassault Aviation. One of the earliest contributions has been Wang's doctoral thesis [WAN 01] in which multicriterion optimization problems in aerodynamics have been treated by Nash games by taking the best advantage of a distributed environment. Nevertheless, note that in some cases of multipoint drag minimization, the lift constraint was introduced by the penalty approach; thus, somewhat artificially, all the criteria were unconstrained and this results in a simplification, because it allows the Nash equilibrium to be sought from an initial point where the functional gradient 
is equal to zero, and the dynamic game develops in a region in which the functional is not very sensitive to parameter changes.

For purpose of illustration, we reproduce here partially the results of a two-point airfoil shape aerodynamic optimization taken from [TAN 07]. The targets are to maximize the lift in a subsonic regime representative of take-off and landing $\left(M_{\infty}=\right.$ $0.2, \alpha=10.8^{\circ}$ ) defining the first point, and concurrently minimize the drag in a transonic flow representative of cruise $\left(M_{\infty}=0.77, \alpha=1^{\circ}\right)$ defining the second point. For both points, the airfoil is assumed to be immersed in a compressible Eulerian flow. Here, both optimizations are treated as inverse problems. A first airfoil shape is associated with the subsonic point; this airfoil is considered satisfactory w.r.t. lift in this regime, and the corresponding pressure distribution along the airfoil is denoted $p_{\text {sub }}$. This airfoil may be the result of a single-point optimization. However, this airfoil should be improved w.r.t. drag at the transonic point. A second airfoil has the opposite characteristics. It is satisfactory w.r.t. drag at the transonic point, and the corresponding pressure distribution along the airfoil is denoted $p_{\text {trans }}$, but not w.r.t. lift at the subsonic point. Then one seeks an airfoil shape that produces in each point a pressure distribution as close as possible to the relevant target profile, $p_{\text {sub }}$ or $p_{\text {trans }}$.

For this, the airfoil boundary $\Gamma_{c}$ is split into two complementary territories $\Gamma_{1}$ and $\Gamma_{2}$, corresponding approximately to the fore and aft regions of the airfoil (see FIG. 4, top). The airfoil is parameterized classically by means of the Hicks-Henne basis functions, and the associated weights are the design variables of the experiment. One such design variable is allocated to either territory depending on the location of the maximum of the corresponding bell-shaped function. In this way, $\Gamma_{1}$ and $\Gamma_{2}$ are associated with specific distinct subsets of the design variables. A trade-off between the two target airfoils is then sought by realizing a Nash equilibrium associated with the following formulation :

$$
\min _{\Gamma_{1}} I_{1}=\int_{\Gamma_{c}}\left(p-p_{\text {sub }}\right)^{2}
$$

(in which it is implicit that the field is calculated in the subsonic conditions of the first point), and

$$
\min _{\Gamma_{2}} I_{2}=\int_{\Gamma_{c}}\left(p-p_{\text {trans }}\right)^{2}
$$

(in which it is implicit that the field is calculated in the transonic conditions of the second point).

Starting with some appropriate initial airfoil, a virtual player performs 5 design cycles to reduce criterion $I_{1}$ by acting only on the subset of the design variables associated with $\Gamma_{1}$, and maintaining the other variables fixed. The optimizer is a steepestdescent-type method based on a functional gradient resulting from discretizing a continuous adjoint equation. In parallel, another virtual player performs 10 design cycles 
a) Split of geometrical parameters

\section{Optimization Strategy}

Player 1

Optimized

Fixed

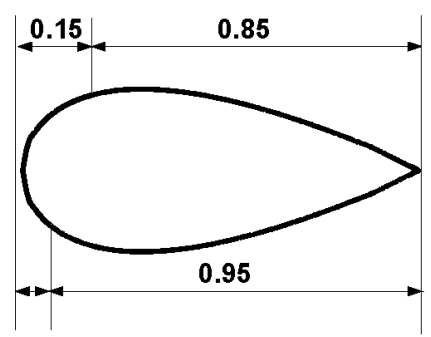

Player 2

Fixed Optimized

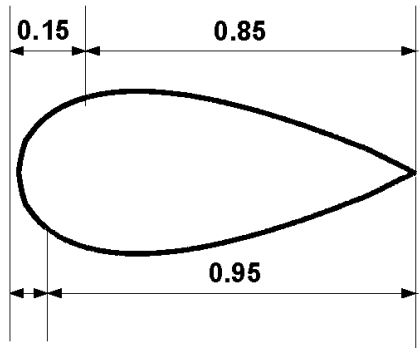

b) Convergence of the two criteria

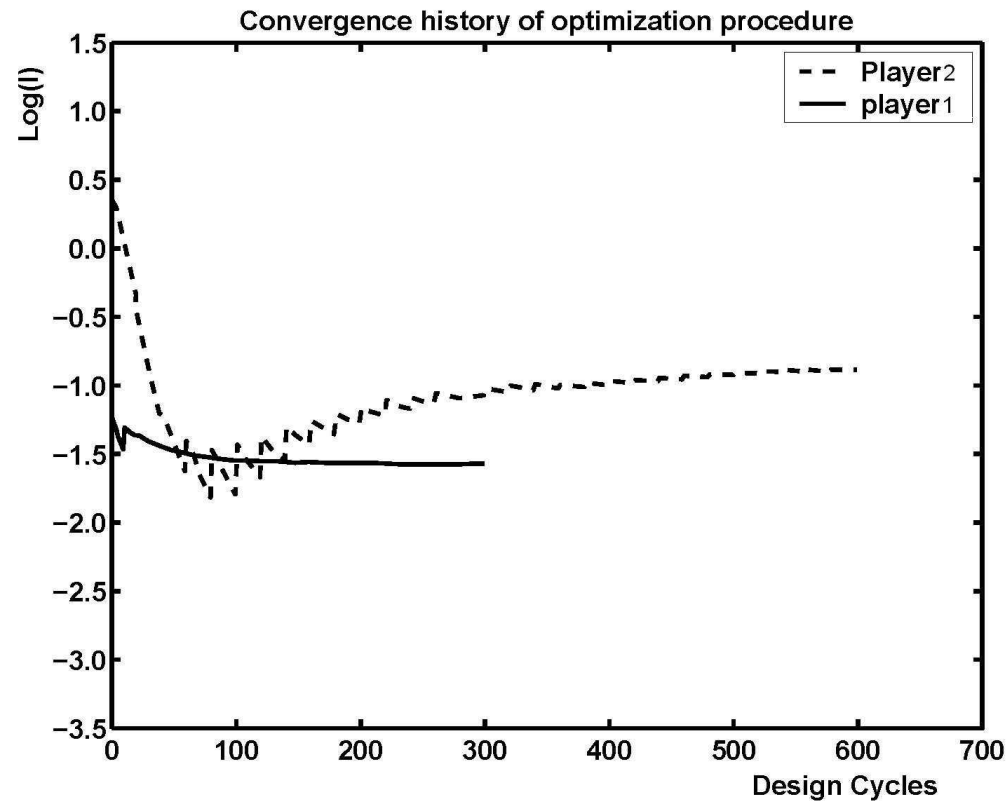

Figure 4. Split of territory and optimization strategy; information exchange every 5 || 10 parallel optimization iterations (top); asymptotic convergence of the two criteria towards a Nash equilibrium (bottom) 
to reduce criterion $I_{2}$ by acting only on the subset of the design variables associated with $\Gamma_{2}$, and maintaining the other variables fixed. Then, both players exchange their best respective subvectors of design variables, and so on until an equilibrium is reached. The iterative convergence of this process is indicated at FIG. 4 (bottom) : both criteria approach a stable asymptote.

FIG. 5 illustrate how the trade-off airfoil shape corresponding to the Nash equilibrium solution compares with the initial and target airfoils, and FIG. 6 provides the pressure distributions over this optimized geometry in the two calculation points.
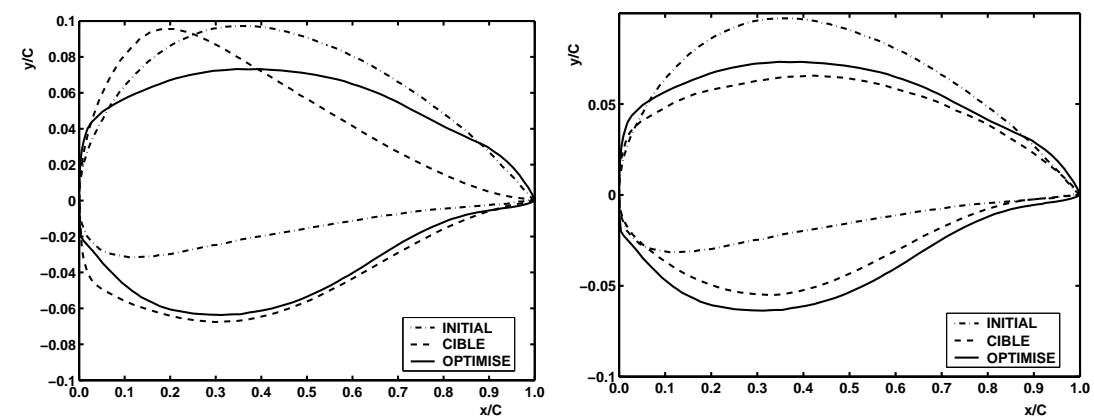

Figure 5. Comparison between the optimized airfoil (solid line) with the initial airfoil and the target airfoil associated with the subsonic conditions of the first point (left), and the target airfoil associated with the transonic conditions of the second point (right)
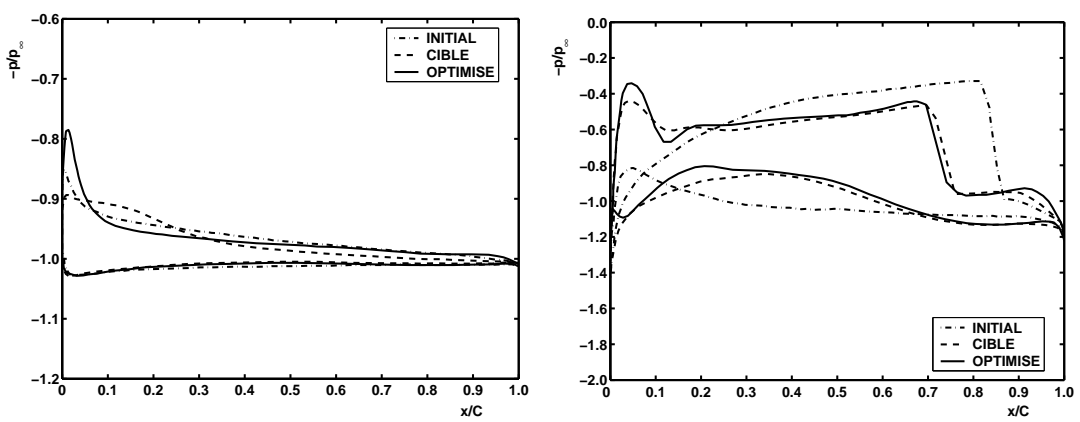

Figure 6. Pressure distributions over the initial, target and optimized (solid line) shapes in the subsonic conditions of the first point (left), and in the transonic conditions of the second point (right) 
Keeping this example in mind, we return now to our general discussion on multiobjective, or multidiscipline optimization. In optimum-shape design in aerodynamics, we are facing two major difficulties.

The first difficulty is related to the fact that only the simulation of a complex flow by a high-fidelity model can provide a realistic evaluation of the aerodynamic coefficients. For instance, the solution of the three-dimensional compressible Euler equations, not so long ago considered as an accomplishment, only provides the wave drag and friction forces are neglected. The computational cost of an accurate evaluation of the aerodynamic functionals is thus very high.

Secondly, by nature transonic flows are only weak solutions to the partialdifferential equations of gasdynamics. As such, they are very sensitive to variations in boundary conditions, such as shape variations. The aerodynamic performance is therefore very fragile, in particular drag, and tolerance margins are small. By coupling aerodynamics with one or more other disciplines in a multidisciplinary optimization, it is imperative to maintain the aerodynamic performance near the optimal level.

We introduce the notion of primary functional w.r.t. which sub-optimality should be maintained, and secondary functional to be reduced under possible constraints.

In our notations, the dimension of the full design space is $N$. A first optimization step is completed in which the sole principal criterion $J_{A}$ is minimized w.r.t. the totality of the design variables, yielding a vector $Y_{A}^{*}$ that realizes, by hypothesis, a local or global minimum of this criterion. It is also assumed that at this point, $K$ scalar constraints $\left(g_{k}=0, k=1,2, \ldots, K\right.$, or more compactly $\left.g=0\right)$ are active. Then, one wishes to conduct a second optimization step, multiobjective and competitive in nature, by establishing a Nash equilibrium between the criteria $J_{A}$ and $J_{B}$. To extend the formulation of the previous experiment, the following more general split of territory is introduced :

$$
Y=Y(U, V)=Y_{A}^{*}+S\left(\begin{array}{c}
U \\
V
\end{array}\right)
$$

where :

$$
U=\left(\begin{array}{c}
u_{1} \\
\vdots \\
u_{N-p}
\end{array}\right), \quad V=\left(\begin{array}{c}
v_{p} \\
\vdots \\
v_{1}
\end{array}\right)
$$

in which $S$ is an adjustable matrix of dimension $N \times N$, referred to as the splitting matrix, and to utilize the subvectors $U\left(U \in \mathbb{R}^{N-p}\right)$ and $V\left(V \in \mathbb{R}^{p}\right)$ as strategies, or territories of two virtual players $A$ and $B$ in charge of the minimization of $J_{A}$ and $J_{B}$ respectively. 
The Nash equilibrium point, if it exists, is denoted $\bar{Y}=Y(\bar{U}, \bar{V})$, and it is associated with the following coupled optimization formulation :

$$
\left\{\begin{array}{l}
\min _{U \in \mathbb{R}^{N-p}} J_{A}[Y(U, \bar{V})] \\
\text { Subject to }: g[Y(U, \bar{V})]=0
\end{array}\right.
$$

and :

$$
\left\{\begin{array}{l}
\min _{V \in \mathbb{R}^{p}} J_{B}[Y(\bar{U}, V)] \\
\text { Subject to : no constraints }
\end{array}\right.
$$

The dimension $p$ of subvector $V$ which controls the subspace of action of player $B$ is adjustable $(p \geq 1)$; however, the dimension $N-p$ of subvector $U$ must be at least equal to 1 , and at least equal to the number $K(K \geq 0)$ of active constraints; this gives the following bounds on $p$ :

$$
1 \leq p \leq N-\max (K, 1)
$$

In the limiting case $(N-p=K)$, in the above Nash game formulation, the minimization of $J_{A}$ under constraints reduces to the adjustment of the $K$ components of subvector $U$ to satisfy the $K$ scalar constraints. This case has been examined in [DÉS 07a]. Hereafter, unless mentioned otherwise, a strict inequality is assumed instead.

In the examples cited above, [WAN 01] and [TAN 07], the split is a partition of the primitive variables, that is, the original components of the design vector $Y$. Our new and more general formulation encompasses this particular case obtained when the splitting matrix is a permutation matrix.

In a parametric shape optimization, the primitive variables are geometrical control parameters, such as the weights put on the different Hicks-Henne basis functions, or the coordinates of control points in a Bézier parameterization. Thus, typically, these variables are associated with specific locations of the optimized geometry. Hence, when the splitting is a permutation, the permutation reflects our intuitive understanding of the dependency of the physical functionals on the geometry, or regions of it. For instance, in the example of FIG. 4, the split was guided by the knowledge that in a transonic flow, the wave drag is the result of the shock intensity and it depends mostly on the delicate design of the geometry on the upper surface near the shock, whereas, in a subsonic flow, the lift is essentially proportional to the airfoil thickness. In his doctoral thesis, Wang [WAN 01] demonstrated that iterations based on choices for the splitting opposite to this physical sense, unsurprisingly, diverge.

These considerations lead us to raise the following question : how should the split be defined in a general and systematic manner to respect the physical sense? In 
particular, if the Nash game is initiated from a viable, physically-relevant solution corresponding to an optimum of the primary criterion $J_{A}$, can near-optimality of this criterion be maintained at equilibrium?

With the formulation of (3), the subspace spanned by the first $N-p$ column vectors of the splitting matrix $S$ can be viewed as the territory assigned to player $A$ in charge of minimizing the primary criterion $J_{A}$, and the subspace spanned by the last $p$ column vectors as the territory assigned to player $B$ in charge of minimizing the secondary criterion $J_{B}$. Thus the above open questions are those of the adequacy of the split of territory. The option which is adopted here consists in making this choice statically (and not adaptively in the course of the dynamic game), at completion of the first step of the procedure in which the primary criterion is minimized alone (possibly under constraints) in full dimension $N$, yielding the optimal design vector $Y_{A}^{*}$, and before any competitive strategy is initiated. Thus the choice is made on the basis of the analysis of the sensitivity of this criterion only. We specifically enforce the following condition : the second step of the optimization procedure, the competitive step, should be such that infinitesimal perturbations of the parameters about $Y_{A}^{*}$ that lie in the subspace identified as the territory of the secondary criterion should cause the least possible degradation of the primary criterion (w.r.t. the minimum achieved at completion of the first step). As a basis for the identification of the optimal splitting, one considers the formal Taylor's expansion of the primary functional to second order about $Y_{A}^{*}$ in the direction of a unit vector $\omega \in \mathbb{R}^{N}$ :

$$
J_{A}\left(Y_{A}^{*}+\varepsilon \omega\right)=J_{A}\left(Y_{A}^{*}\right)+\varepsilon \nabla J_{A}^{*} \cdot \omega+\frac{\varepsilon^{2}}{2} \omega \cdot H_{A}^{*} \omega+O\left(\varepsilon^{3}\right)
$$

Our goal is to propose a sensible splitting associated with the definition of a vector basis $\left\{\omega^{k}\right\}(k=1, \ldots, N)$. To fix the ideas, let us assume that the first few elements, $\left\{\omega^{k}\right\}(k=1,2, \ldots)$, of the basis are dedicated to player $\mathrm{A}$ in charge of reducing the primary criterion $J_{A}$, and inversely, the tail elements, $\left\{\omega^{k}\right\}$ ( $\left.k=N, N-1 \ldots\right)$, to player B in charge of reducing the secondary criterion $J_{B}$. Note that the direction of maximum sensitivity of the primary criterion $J_{A}$, or steepest-descent direction, is given by the gradient, $\nabla J_{A}^{*}$ at $Y=Y_{A}^{*}$. Thus, the following two conditions should be satisfied by the basis :

1) the first few elements should span the gradient, $\nabla J_{A}^{*}$;

2) inversely, the difference $\left|J_{A}\left(Y_{A}^{*}+\varepsilon \omega\right)-J_{A}\left(Y_{A}^{*}\right)\right|$, when $\varepsilon$ is small and fixed, should be as small as possible when $\omega$ is a tail element of the basis.

At $Y=Y_{A}^{*}$, the optimality conditions imply that the gradient $\nabla J_{A}^{*}$ is a linear combination of the $K$ active constraint gradients, the coefficients being the Lagrange multipliers. Thus a way to achieve the first condition is to enforce that the first $K$ elements of the basis have the same span as the gradients of the $K$ active constraints. For this, one requires that $\left\{\omega^{k}\right\}(k=1,2, \ldots, K)$ be the result of applying the Gram-Schmidt 
orthogonalization process to the constraint gradients $\left\{\nabla g_{k}^{*}\right\}(k=1,2, \ldots, K)$. Then, let $P$ be the following projection matrix :

$$
P=I-\sum_{k=1}^{K}\left[\omega^{k}\right]\left[\omega^{k}\right]^{T}
$$

where $\left[\omega^{k}\right]$ denotes the column-vector matrix made of the components of vector $\omega^{k}$, and consider the following real-symmetric matrix :

$$
H_{A}^{\prime}=P H_{A}^{*} P
$$

We claim that the eigenvectors of the matrix $H_{A}^{\prime}$, ordered appropriately, constitute the best choice.

First, these eigenvectors contain the null space of the projection matrix $P$, that is, $\left\{\omega^{k}\right\}(k=1,2, \ldots, K)$. Thus the first condition is satisfied simply if the ordering is such that these vectors appear first.

Second, the basis is orthogonal; hence the tail elements are orthogonal to the first $K$, and to $\nabla J_{A}^{*}$ as a consequence of the first condition. Thus, for $\omega=\omega^{k}(k \geq K+1)$, the principal term in the expansion of the difference, $\left|J_{A}\left(Y_{A}^{*}+\varepsilon \omega\right)-J_{A}\left(Y_{A}^{*}\right)\right|$ is the quadratic term. This term, including the absolute value, reduces to the Rayleigh quotient associated with the matrix $H_{A}^{\prime}$ (assuming positive-definiteness), and the classical characterization of eigenvectors, here by decreasing eigenvalue, holds.

Starting from the above observations, the following theorem, taken from [DÉS 07a], exploits this basic principle and draws certain additional consequences related to the Nash game. It is assumed that the two criteria $J_{A}$ and $J_{B}$ are strictly positive and such that :

$$
J_{A}^{*}=J_{A}\left(Y_{A}^{*}\right)>0, \quad J_{B}^{*}=J_{B}\left(Y_{A}^{*}\right)>0
$$

If necessary the problem can easily be reformulated to meet these requirements.

\section{Theorem 1}

Let $N, p$ and $K$ be positive integers such that :

$$
1 \leq p \leq N-\max (K, 1)
$$

Let $J_{A}, J_{B}$ and, if $K \geq 1,\left\{g_{k}\right\}(1 \leq k \leq K)$, be $K+2$ smooth real-valued functions of the vector $Y \in \mathbb{R}^{N}$. Assume that $J_{A}$ and $J_{B}$ are positive, and consider the following primary optimization problem,

$$
\min _{Y \in \mathbb{R}^{N}} J_{A}(Y)
$$


that is either unconstrained ( $K=0$ ), or subject to the following $K$ equality constraints :

$$
g(Y)=\left(g_{1}, g_{2}, \ldots, g_{K}\right)^{T}=0
$$

Assume that the above minimization problem admits a local or global solution at a point $Y_{A}^{*} \in \mathbb{R}^{N}$ at which $J_{A}^{*}=J_{A}\left(Y_{A}^{*}\right)>0$ and $J_{B}^{*}=J_{B}\left(Y_{A}^{*}\right)>0$, and let $H_{A}^{*}$ denote the Hessian matrix of the criterion $J_{A}$ at $Y=Y_{A}^{*}$.

If $K=0$, let $P=I$ and $H_{A}^{\prime}=H_{A}^{*}$; otherwise, assume that the constraint gradients, $\left\{\nabla g_{k}^{*}\right\}(1 \leq k \leq K)$, are linearly independent and apply the Gram-Schmidt orthogonalization process to the constraint gradients, and let $\left\{\omega^{k}\right\}(1 \leq k \leq K)$ be the resulting orthonormal vectors. Let $P$ be the matrix associated with the projection operator onto the $K$-dimensional subspace tangent to the hyper-surfaces $g_{k}=0$ $(1 \leq k \leq K)$ at $Y=Y_{A}^{*}$,

$$
P=I-\sum_{k=1}^{K}\left[\omega^{k}\right]\left[\omega^{k}\right]^{T}
$$

where $\left[\omega^{k}\right]$ denotes the column-vector matrix made of the components of vector $\omega^{k}$, and consider the following real-symmetric matrix :

$$
H_{A}^{\prime}=P H_{A}^{*} P
$$

Let $\Omega$ be an orthogonal matrix whose column-vectors are normalized eigenvectors of the matrix $H_{A}^{\prime}$ organized in such a way that the first $K$ are precisely $\left\{\omega^{k}\right\}(1 \leq k \leq$ $K)$, and the subsequent $N-K$ are arranged by decreasing order of the eigenvalue

$$
h_{k}^{\prime}=\omega^{k} \cdot H_{A}^{\prime} \omega^{k}=\omega^{k} \cdot H_{A}^{*} \omega^{k} \quad(K+1 \leq k \leq N)
$$

Consider the splitting of parameters defined by :

$$
Y=Y_{A}^{*}+\Omega\left(\begin{array}{c}
U \\
V
\end{array}\right), \quad U=\left(\begin{array}{c}
u_{1} \\
\vdots \\
u_{N-p}
\end{array}\right), \quad V=\left(\begin{array}{c}
v_{p} \\
\vdots \\
v_{1}
\end{array}\right)
$$

Let $\varepsilon$ be a small positive parameter $(0 \leq \varepsilon \leq 1)$, and let $\bar{Y}_{\varepsilon}$ denote the Nash equilibrium point associated with the concurrent optimization problem :

$$
\left\{\begin{array} { l } 
{ \operatorname { m i n } _ { U \in \mathbb { R } ^ { N - p } } J _ { A } } \\
{ \text { Subject to } : g = 0 }
\end{array} \text { and } \quad \left\{\begin{array}{l}
\min _{V \in \mathbb{R}^{p}} J_{A B} \\
\text { Subject to : no constraints }
\end{array}\right.\right.
$$

in which again the constraint $g=0$ is not considered when $K=0$, and

$$
J_{A B}:=\frac{J_{A}}{J_{A}^{*}}+\varepsilon\left(\theta \frac{J_{B}}{J_{B}^{*}}-\frac{J_{A}}{J_{A}^{*}}\right)
$$


where $\theta$ is a strictly-positive relaxation parameter $(\theta<1$ : under-relaxation; $\theta>1$ : over-relaxation).

\section{Then :}

- [Optimality of orthogonal decomposition] If the matrix $H_{A}^{\prime}$ is positive semidefinite, which is the case in particular if the primary problem is unconstrained $(K=0)$, or if it is subject to linear equality constraints, its eigenvalues have the following structure :

$$
h_{1}^{\prime}=h_{2}^{\prime}=\ldots=h_{K}^{\prime}=0 \quad h_{K+1}^{\prime} \geq h_{K+2}^{\prime} \geq \ldots \geq h_{N}^{\prime} \geq 0
$$

and the tail associated eigenvectors $\left\{\omega^{k}\right\}(K+1 \leq k \leq N)$ have the following variational characterization :

$$
\begin{aligned}
\omega^{N} & =\operatorname{Argmin}_{\omega}\left|\omega . H_{A}^{*} \omega\right| \text { s.t. }\|\omega\|=1 \text { and } \omega \perp\left\{\omega^{1}, \omega^{2}, \ldots, \omega^{K}\right\} \\
\omega^{N-1} & =\operatorname{Argmin}_{\omega}\left|\omega . H_{A}^{*} \omega\right| \text { s.t. }\|\omega\|=1 \text { and } \omega \perp\left\{\omega^{1}, \omega^{2}, \ldots, \omega^{K}, \omega^{N}\right\} \\
\omega^{N-2} & =\operatorname{Argmin}_{\omega}\left|\omega . H_{A}^{*} \omega\right| \text { s.t. }\|\omega\|=1 \text { and } \omega \perp\left\{\omega^{1}, \omega^{2}, \ldots, \omega^{K}, \omega^{N}, \omega^{N-1}\right\} \\
& \vdots
\end{aligned}
$$

- [Preservation of optimum point as a Nash equilibrium] For $\varepsilon=0$, a Nash equilibrium point exists and it is :

$$
\bar{Y}_{0}=Y_{A}^{*}
$$

- [Robustness of original design] If the Nash equilibrium point $\bar{Y}_{\varepsilon}$ exists for $\varepsilon>0$ and sufficiently small, and if it depends smoothly on this parameter, the functions :

$$
j_{A}(\varepsilon)=J_{A}\left(\bar{Y}_{\varepsilon}\right), \quad j_{A B}(\varepsilon)=J_{A B}\left(\bar{Y}_{\varepsilon}\right)
$$

are such that :

$$
\begin{gathered}
j_{A}^{\prime}(0)=0 \\
j_{A B}^{\prime}(0)=\theta-1 \leq 0
\end{gathered}
$$

and

$$
\begin{gathered}
j_{A}(\varepsilon)=J_{A}^{*}+O\left(\varepsilon^{2}\right) \\
j_{A B}(\varepsilon)=1+(\theta-1) \varepsilon+O\left(\varepsilon^{2}\right)
\end{gathered}
$$

- In case of linear equality constraints, the Nash equilibrium point satisfies identically:

$$
\begin{gathered}
u_{k}(\varepsilon)=0 \quad(1 \leq k \leq K) \\
\bar{Y}_{\varepsilon}=Y_{A}^{*}+\sum_{k=K+1}^{N-p} u_{k}(\varepsilon) \omega^{k}+\sum_{j=1}^{p} v_{j}(\varepsilon) \omega^{N+1-j}
\end{gathered}
$$


- For $K=1$ and $p=N-1$, the Nash equilibrium point $\bar{Y}_{\varepsilon}$ is Pareto optimal.

We have seen already why the proposed basis of eigenvectors is optimal for the problematics raised by the case of a preponderant or fragile discipline, in relation with the performance of the Nash equilibrium solution; shortly speaking, the spliiting is such that a minimal degradation of $J_{A}$ is caused by the reduction of $J_{B}$. Another aspect is the existence itself of this equilibrium. With respect to this, and without entering all the details of the full proof, given in [DÉS 07a], let us examine the mechanism by which the present choice of territory splitting also permits to guarantee the preservation of initial optimum point of discipline $A$ alone, $Y_{A}^{*}$, as a Nash equilibrium of the above formulation for $\varepsilon=0$, as stated in (23).

For $\varepsilon=0$, let the criterion $J_{A}=J$ for notational simplicity. The criteria $J_{A B}$ and $J$ are functionally proportional, and so are their gradients. We wish to establish that $U=V=0$ indeed corresponds to a Nash equilibrium.

On one side, for fixed $V=0$, the subvector $U=0$ indeed realizes the minimum of $J_{A}=J$ subject to the constraint $g=0$, because the optimization of $U$ is equivalent to the minimization of $J_{A}$ in a subset that contains the solution $Y_{A}^{*}$ of the minimization in the full design space.

On the other side, for fixed $U=0$, the (unconstrained) derivative of $J_{A B}$ w.r.t. $V$ is proportional to :

$$
\frac{\partial J}{\partial V}=\nabla J \cdot \frac{\partial Y}{\partial V}=\nabla J_{A}^{*} \cdot \frac{\partial Y}{\partial V}=0
$$

because, by construction of the split, the vector $\frac{\partial Y}{\partial V}$ is a linear combination of the tail elements of the eigenvector basis, and these are orthogonal to the first $K$ elements, and those span a subspace containing $\nabla J_{A}^{*}$. Hence, for fixed $U=0$, the unconstrained criterion $J_{A B} \sim J$ is also stationary w.r.t. subvector $V$ at $V=0$.

In summary, this theorem establishes two main achievements related to the Nash equilibrium solution :

- A potential performance result : it permits to identify abstractly an orthogonal decomposition of the parameter-space that is such that for given dimension $p$ ( $p \leq N-\max (K, 1)$ ), the tail $p$ vectors of the basis correspond to the directions of least variation of the primary functional $J_{A}$ from its minimum value under possible equality constraints; in this sense, these eigenvectors span the subspace of dimension $p$ in which the primary functional is the most insensitive to the small variations in the design vector that will be made, in a second phase of optimization, to reduce a secondary functional, $J_{B}$;

- An existence result : a procedure involving a continuation parameter $\varepsilon(0 \leq \varepsilon \leq$ 1) has been set up permitting to introduce gradually and smoothly the secondary functional $J_{B}$ in competition with the primary functional $J_{A}$ in a Nash game; for $\varepsilon=0$, it 
is established that the original optimal solution $Y_{A}^{*}$ is a Nash equilibrium point of the initially-trivial game formulation; consequently, by continuity, the Nash equilibrium solution exists, at least for $\varepsilon$ sufficiently small. Another parameter $\theta$ appears in the formulation; it allows under or over-relaxation of the process; if $\theta<1$, the auxiliary criterion $J_{A B}$ at the Nash equilibrium point $\bar{Y}_{\varepsilon}$ decreases when $\varepsilon$ increases, but remains sufficiently small; since $\bar{Y}_{0}=Y_{A}^{*}$, the locus of $\bar{Y}_{\varepsilon}$ as $\varepsilon$ varies is viewed as a continuation of the original optimum point of the primary functional alone.

The construction of the orthogonal basis is made at full convergence of the minimization of the primary functional by diagonalization of the Hessian matrix restricted to the subspace tangent to the hypersurfaces representing the active constraints. To identify this tangent subspace, a Gram-Schmidt orthogonalization process is applied to the constraint gradients. In practice, the Hessian can be calculated exactly either formally or by automatic differentiation; otherwise, an approximation can be made by differentiating a metamodel for the primary functional and constraints valid in a neighborhood of the optimal solution $Y_{A}^{*}$. This metamodel can be, for example, an artificial neural network or a Kriging model (see for instance [CHA 07] [DUV 07]).

We close this section by emphasizing again the merit of our formulation, when equality constraints are active, to remain consistent with the single-criterion minimization of the primary functional alone at the initial point $\varepsilon=0$ of the continuation procedure $\left(\bar{Y}_{0}=Y_{A}^{*}\right)$. This nontrivial property usually does not hold when the split is made over the primitive variables as formerly proposed in [WAN 01] [TAN 07], unless the constraints are treated by the penalty approach. The variations in the primary functional are initially second-order in $\varepsilon$; thus the new formulation permits to identify smoothly the locus of Nash equilibrium solutions as $\varepsilon$ varies, by an algorithm whose iterative convergence is facilitated by this robustness property, since the potential antagonism between the two criteria can be introduced as smoothly as necessary by small enough steps in the continuation parameter $\varepsilon$.

\subsection{Aerostructural shape optimization of a business jet wing}

In order to illustrate the influence of the split of territory on the result of a practical two-discipline optimization, the main results achieved by B. Abou El Majd in his doctoral thesis [Abo 07] concerning a case of aerostructural shape optimization of a business jet wing are reproduced here. In his thesis, a detailed description of a number of algorithmic variants, including those whose formulation relies on a hierarchical Stackelberg game (instead of a symmetrical Nash game), have been tested and analyzed systematically.

Aerodynamics is treated as the preponderant discipline; it will also reveal to be a fragile discipline. The flow about the wing is computed by a finite-volume simulation of the three-dimensional Euler equations. The method handles unstructured grids by 
the construction of a medians-based dual finite-volume mesh, and employs a Roetype upwind solver. The computation yields the wave drag coefficient, $C_{D}$, as well as other aerodynamic coefficients, such as lift, $C_{L}$. The simulation point is transonic $\left(M_{\infty}=0.83, \alpha=2^{\circ}\right)$. The primary objective is to minimize the drag coefficient augmented by a penalty term which is active when a minimal lift coefficient constraint is violated. Thus, the primary criterion admits the following expression :

$$
J_{A}=\frac{C_{D}}{C_{D_{0}}}+10^{4} \max \left(0,1-\frac{C_{L}}{C_{L_{0}}}\right)
$$

in which the reference quantities, indicated by the subscript ${ }_{0}$ correspond to an initial geometry defined by an initial three-dimensional unstructured grid about the wing.

Throughout the optimization process, the geometry is iteratively modified according to the so-called Free-Form Deformation (FFD) method which originates from computer vision, and was proposed in the context of an aerostructural design loop by Samareh [SAM 00]. In this approach, a formula is given a priori, in a closed form involving adjustable parameters, to a three-dimensional deformation field, formally and independently of the discrete or continuous representation of the geometry itself, here an unstructured volume mesh. By construction, the deformation field is made to be smooth and equal to zero outside of a support, which is usually a bounding box of simple shape whose boundaries are not made in general of meshpoints. At a given optimization iteration, the deformation field is redefined and applied to the meshpoints lying inside the support, thus permitting an update of the surface meshpoints, but also of meshpoints in the computed volume in the vicinity of the optimized surface. In this way, an initial unstructured volume mesh evolves according to a deformation defined explicitly in terms of the FFD parameters. These parameters are taken to be the design variables of the optimization loop and they are updated here according to the NelderMead [NEL 65] simplex method to reduce the above criterion $J_{A}$.

This procedure results in a simple and fairly robust iterative algorithm. In our experience, this procedure is less subject to mesh overlapping than a volume mesh reconstruction from the displacement of the boundary meshpoints by a pseudo-elasticity equation, such as the spring method.

In our experiments, a system of generalized coordinates $(\xi, \eta, \zeta)$ is defined and corresponds to longitudinal, vertical and span-wise directions. When the bounding box is a parallelepiped, the transfinite interpolation of the Cartesian coordinates suffices to define these transformed coordinates throughout the box. Then, the deformation field is defined as a linear combination of products of three Bernstein polynomials of these coordinates. Precisely, an arbitrary point $q$ is given the following displacement $\Delta q$ :

$$
\Delta q=\sum_{i=0}^{n_{i}} \sum_{j=0}^{n_{j}} \sum_{k=0}^{n_{k}} B_{n_{i}}^{i}\left(\xi_{q}\right) B_{n_{j}}^{j}\left(\eta_{q}\right) B_{n_{k}}^{k}\left(\zeta_{q}\right) \Delta P_{i j k}
$$


in which, for the $k$ th Bernstein polynomial of degree $n$,

$$
B_{n}^{k}(t)=\frac{n !}{k !(n-k) !} t^{k}(1-t)^{n-k}
$$

The degrees of the parameterization in the three physical directions, $\left(n_{i}, n_{j}, n_{k}\right)$, are fixed, and the vector-valued weighting coefficients $\left\{\Delta P_{i j k}\right\}\left(0 \leq i \leq n_{i}\right.$, $0 \leq j \leq n_{j}, 0 \leq k \leq n_{k}$ ) are the design variables of the optimization. Such a geometrical parameterization generalizes the Bézier curve formula, and combined with the classical degree-elevation process, it facilitates the construction of multilevel optimization algorithms inspired by multigrid methods. More details on this method, and more examples of application can be found in [DÉS 07b] [Abo 08].

The deformation field was chosen to be linear span-wise from root to tip $\left(n_{k}=1\right)$. Additionally, the leading and trailing edges, and the eight vertices of the bounding box were fixed throughout the optimization. Finally, only vertical displacements were considered for simplicity.

In a first experiment (see FIG. 7), 6 control points at the root and at the tip were considered, for a total of 12 degrees of freedom.

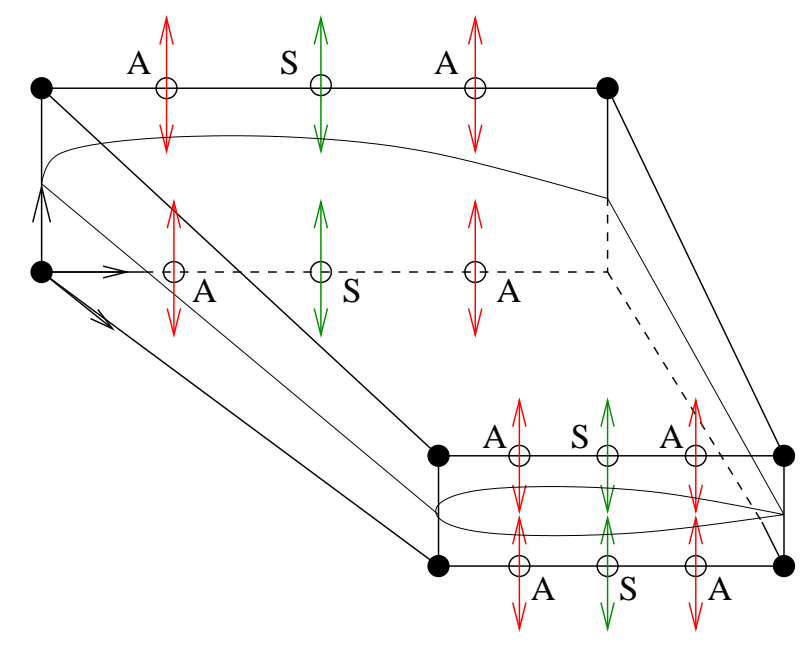

Figure 7. Aerostructural shape optimization of a business jet wing; first split of territory, according to the primitive variables : parameters marked A are associated to aerodynamics, and those marked $S$ to structural design.

In order to define an exercise in which the wing shape is optimized w.r.t. two disciplines, aerodynamics and structural design, that share a common set of design 
variables, the wing structure was treated as a thin shell which deforms under the load of aerodynamic forces. The distribution of stresses over the shell has been calculated by linear-elasticity, using a code of the public domain, ASTER developed by Electricité de France $(E D F)$.

The four degrees of freedom located at mid-chord (at root and tip, over the upper and lower surfaces), marked S on FIG. 7, were assigned to a player in charge of minimizing the following secondary criterion :

$$
\begin{aligned}
J_{B}=J_{S}= & \iint_{S}\|\sigma \cdot n\| d S \\
& +K_{1} \max \left(0,1-\frac{V}{V_{A}}\right)+K_{2} \max \left(0, \frac{S}{S_{A}}-1\right)
\end{aligned}
$$

in which $\sigma$ is the stress tensor, $S_{A}$ and $V_{A}$ are the wing outer surface and volume at convergence of the purely-aerodynamic optimization, and $K_{1}$ and $K_{2}$ and penalty constants. By the reduction of this criterion, one expects a more uniform distribution of the load, and thus a more robust structure.

The remaining 8 degrees of freedom, marked A on FIG. 7, were assigned to a player A in charge of minimizing the primary criterion, $J_{A}$.

It was possible to achieve a Nash equilibrium solution associated with the above split of the primitive variables, as indicated on FIG. 8 which displays the convergence history of the aerodynamic and structural criteria. The sudden and occasional peaks correspond to iterations at which the constraint on lift is violated. The simplex method accommodates to this situation by discarding the point. Evidently, a stable Nash equilibrium is reached eventually.

Regrettably, this Nash-equilibrium configuration is totally unacceptable from a physical standpoint. The drag coefficient has doubled. The wing shape presents oscillations and the flow has been profoundly disrupted as indicated by the Mach number field (see FIG. 9).

By this first experiment, we emphasize that even in case of convergence of the Nash equilibrium, the achieved configuration makes sense only if the split of variables is physically relevant.

In a second experiment, the number of design variables was reduced to 8 by considering a deformation field, only vertical and associated with the polynomial degrees $(3,1,1)$ along the longitudinal, vertical and span-wise directions. After a number of unsuccessful trials, a certain split of the primitive variables yielded acceptable results. The split corresponds to assign the 4 degrees of freedom at the root to player $\mathrm{S}(=\mathrm{B})$ in charge of reducing the structural criterion, and the other 4 , at the tip, to player A in charge of reducing the aerodynamic criterion (see FIG. 10). 
26 Multidisciplinary Design Optimization in Computational Mechanics

a) $\operatorname{Drag}\left(J_{A}\right)$

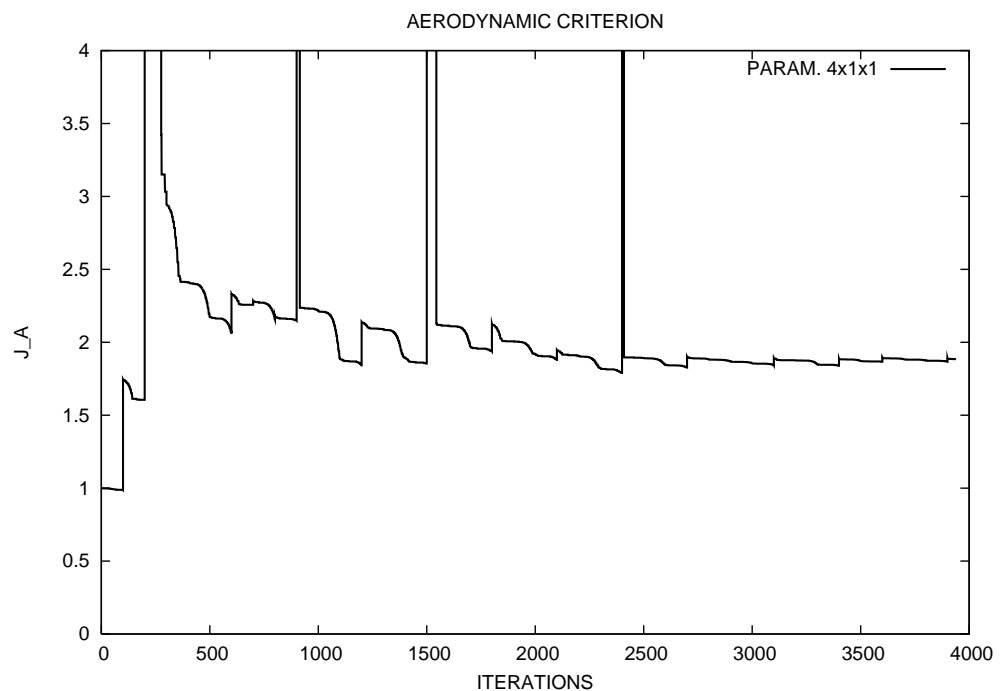

b) Stress integral $\left(J_{S}\right)$

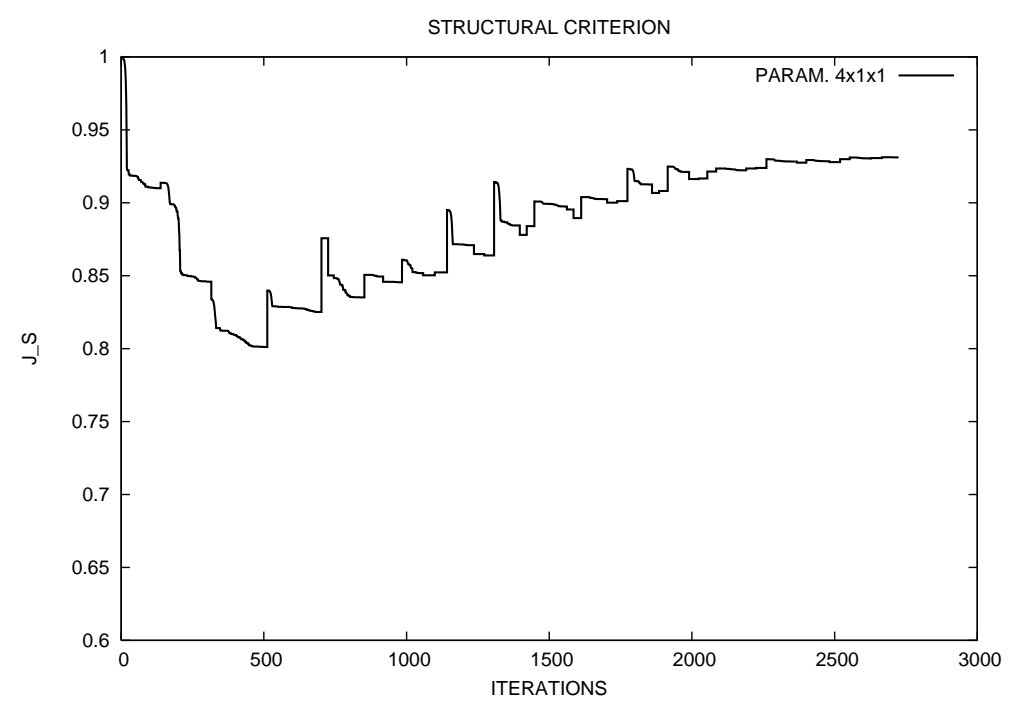

Figure 8. Aerostructural shape optimization of a business jet wing; first split of territory, according to the primitive variables : convergence history of the aerodynamic and structural criteria. 
a) Purely aerodynamic optimization

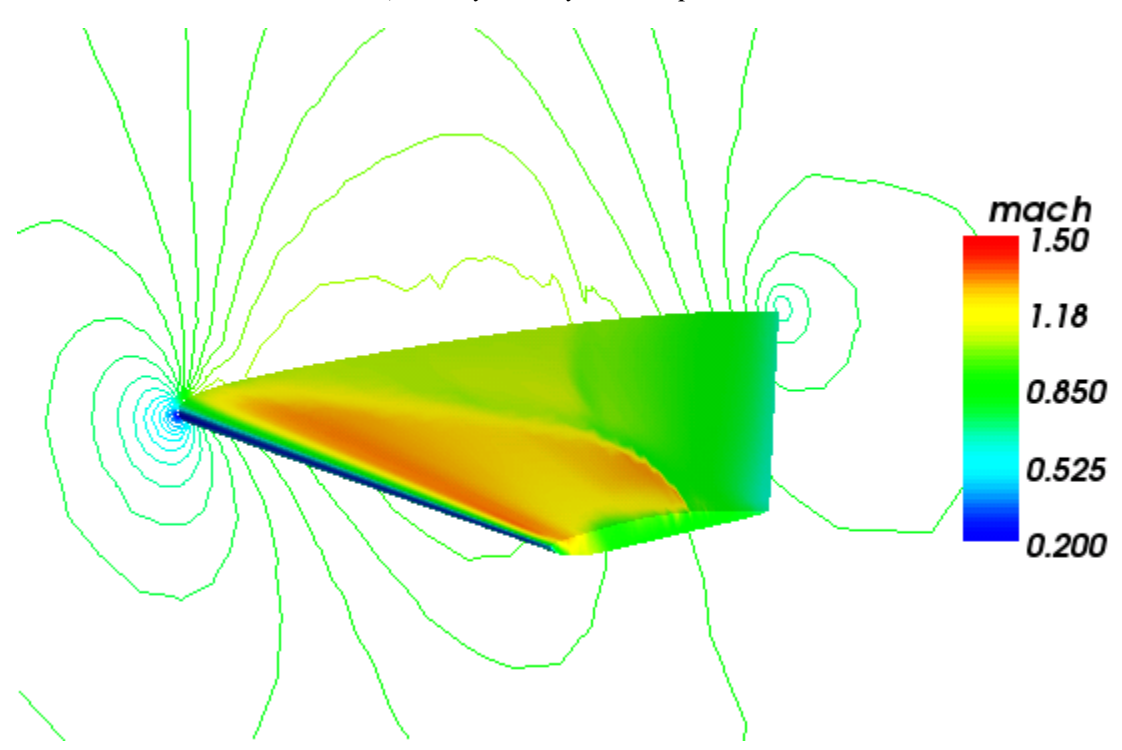

b) Nash equilibrium (unacceptable)

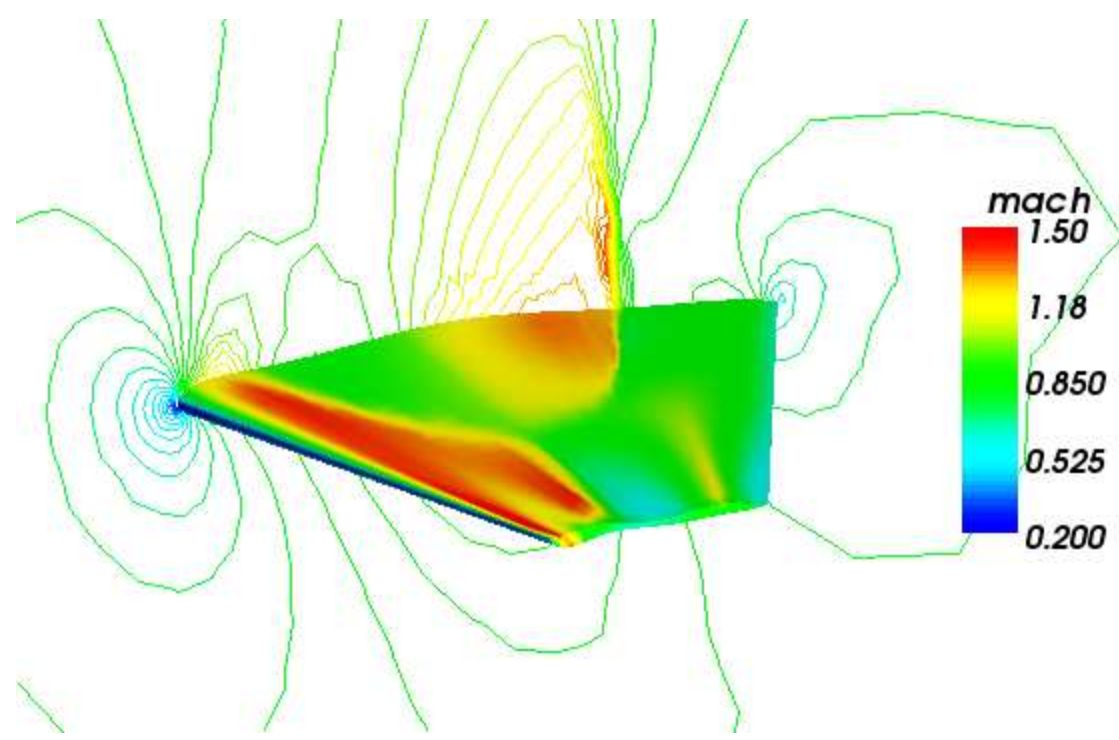

Figure 9. Aerostructural shape optimization of a business jet wing; first split of territory, according to the primitive variables : shape and Mach number field: a) purely aerodynamic optimization, and b) Nash equilibrium. 


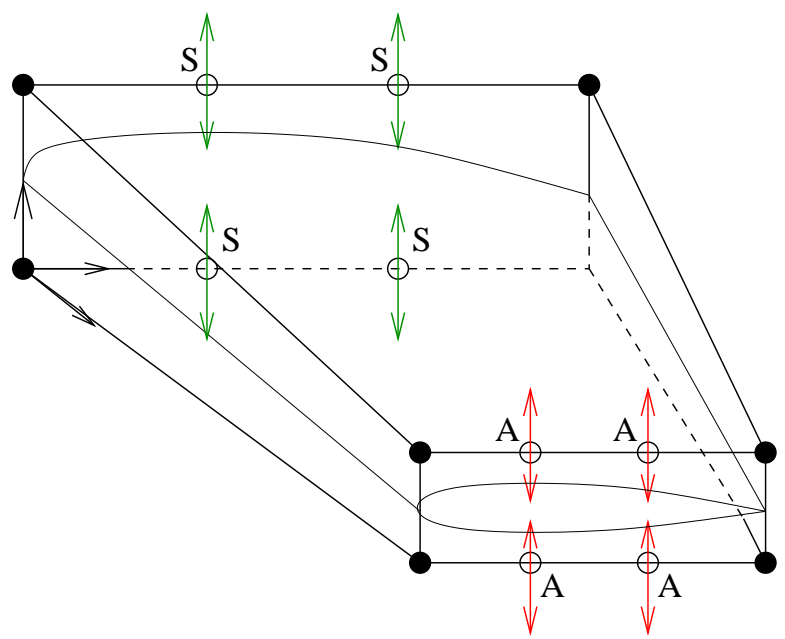

Figure 10. Aerostructural shape optimization of a business jet wing; second split of territory, according to the primitive variables : parameters marked A are associated to aerodynamics, and those marked $S$ to structural design.

The convergence history of the two criteria in the dynamic game corresponding to this new split of design variables is indicated at FIG. 11. The aerodynamic criterion is subject to numerous jumps due to the violation of the constraint on lift, but, as mentioned above, the simplex method accommodates to this. This phase of optimization is interrupted, somewhat arbitrarily after some 380 structural design steps; strictly speaking, convergence is not achieved, but the solution satisfactory since it realizes a visible improvement of the structural criterion of about $5 \%$, while the aerodynamic criterion has been increased of about the same percentage (only).

The cross sections at root, mid-span and wing tip corresponding to the initial and optimized shapes are represented on FIG. 12. It appears that the structural control parameters tend to round out very slightly the root cross section for a better load distribution. This trend augments the drag, but here in proportions still acceptable, because the process was interrupted after a variation of $5 \%$ of each criterion. In fact, at this level of only partial convergence, the shape variations are still very small in amplitude because the coupling mechanism realized by the dynamic game is very stringent. Additionally, our a priori knowledge of the flow led us to locate the aerodynamic control parameters near the wing tip in the vicinity of the most sensitive region of the shock wave. Thus, this experiment does not reflect a blind split of variables, but instead one that was anticipated to be physically sound; and this was confirmed.

In the third experiment, the split of variables based on the proposed orthogonal decomposition of the restricted Hessian was implemented. Once the optimum of 


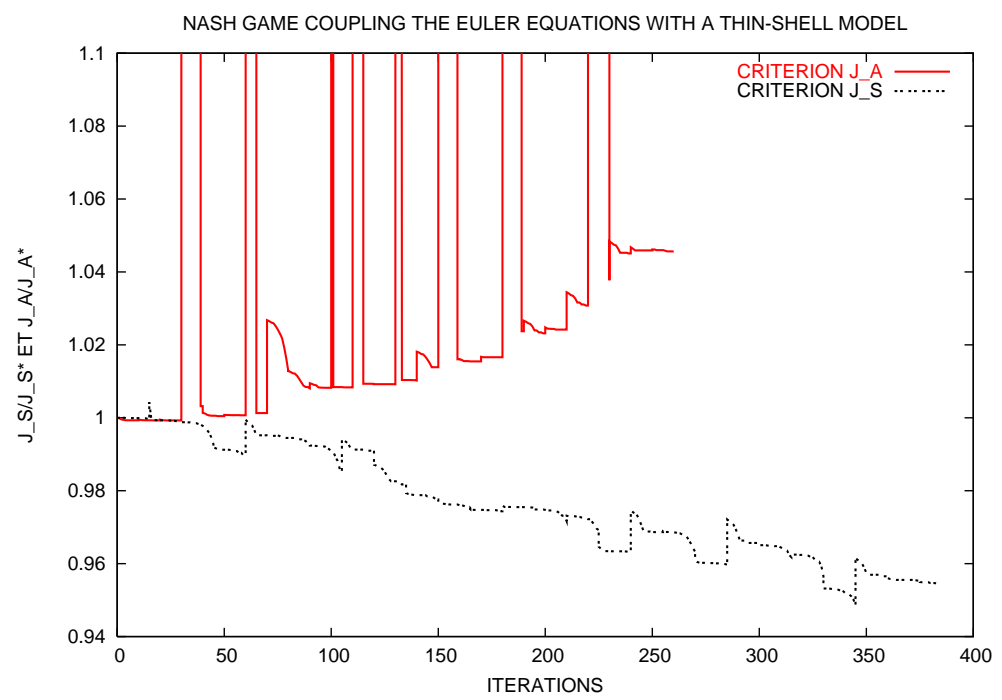

Figure 11. Aerostructural shape optimization of a business jet wing; second split of territory, according to the primitive variables : convergence history of the two criteria

aerodynamics alone has been found at $Y=Y_{A}^{*}$, a number of independent simulations corresponding to design vectors close to $Y_{A}^{*}$ have been made to set up a database to model the behavior of the primary criterion $J_{A}$ in terms of $Y$ by an RBF neural network [CHA 07] [DUV 07]. This metamodel was then used to approximate the gradient of $C_{D}$, the primary criterion to be minimized, the gradient of $C_{L}$, the constrained quantity, and the Hessian of $C_{D}$ to form the restricted Hessian matrix. After diagonalization, the corresponding eigenvectors have been sorted by decreasing order of the associated eigenvalue, and split evenly in two subsets of four. Those associated with the four largest eigenvalues have been assigned to player A in charge of aerodynamics, and the remaining four to player $\mathrm{S}(=\mathrm{B})$ in charge of reducing the criterion of structural design.

The proposed eigensplit led to a new dynamic Nash game, whose convergence history is indicated on FIG. 13. The process was continued to a stage of convergence similar to previously in terms of coupling iterations. However, a notably superior performance was achieved : while the aerodynamic criterion was here only degraded of $3 \%$, the structural criterion was reduced of $8 \%$; equivalently, at equal stage of drag degradation, the improvement on the structural criterion is nearly three times larger. Note how the envelopes of the two curves are apparently initially tangent to the horizontal axis, a hint that in this formulation, the initial point is a robust design. 
30 Multidisciplinary Design Optimization in Computational Mechanics

\section{a) Root}

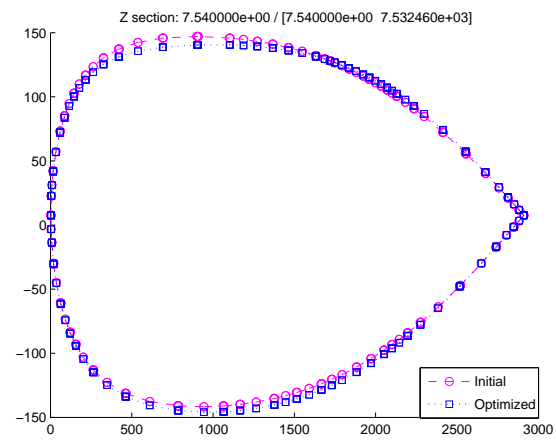

b) Mid-span

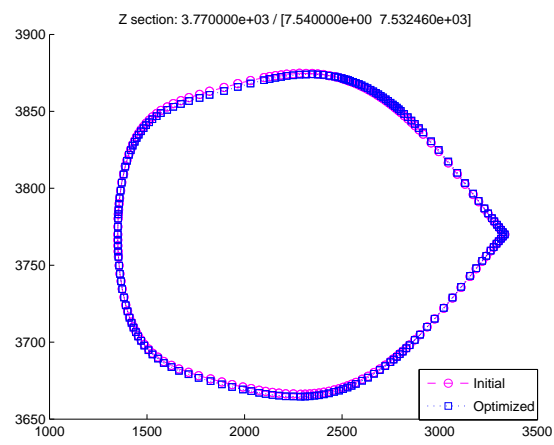

c) Wing tip

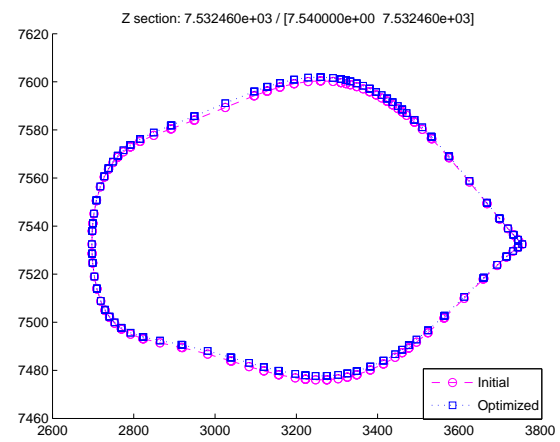

Figure 12. Aerostructural shape optimization of a business jet wing; second split of territory, according to the primitive variables : cross-section variations at a) root, b) mid-span, and c) wing tip. 


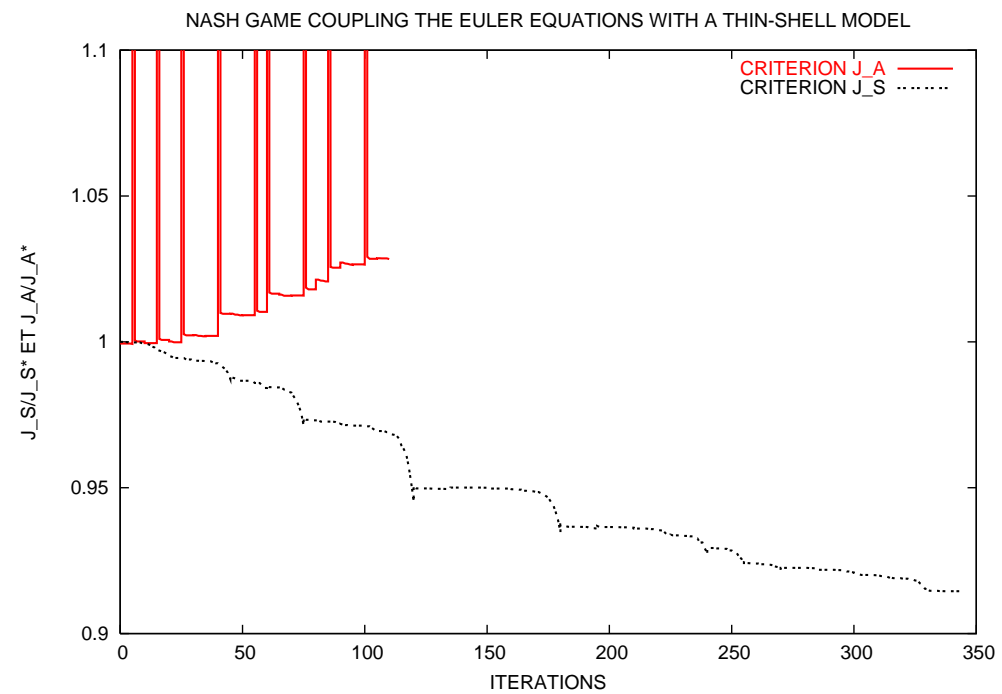

Figure 13. Aerostructural shape optimization of a business jet wing; split of variables according to the orthogonal decomposition; convergence history of the two criteria (after 50 couplings).

FIG. 14 indicates the evolution of cross-sections at root, mid-chord and wing tip. It clearly appears from this figure that the shape variations are of larger amplitude in this experiment than before, in the previous two experiments, but more distinctly located, as for example, on the lower surface of the wing at the root. Thus a wider operational territory for the secondary criterion is identified to cause a small and acceptable degradation only of the first criterion.

The split based on the orthogonal decomposition has permitted us to identify by a blind and automatic procedure, a set of structural parameters for which variations of larger amplitude, mostly visible on the lower surface of the wing, are possible without excessively affecting the shape in the critical region of the shock wave. Consequently, the principal characteristics of the flow are preserved, as indicated on FIG. 15 which shows that the Mach number field has not been much altered from that obtained by pure aerodynamic optimization.

Thus, in conclusion, a significant reduction of $8 \%$ of the structural criterion was realized while maintaining the flowfield configuration close to optimality (drag increase $<3 \%$ ), by an automatic procedure of orthogonal decomposition of the parameter space. 
32 Multidisciplinary Design Optimization in Computational Mechanics

a) Root

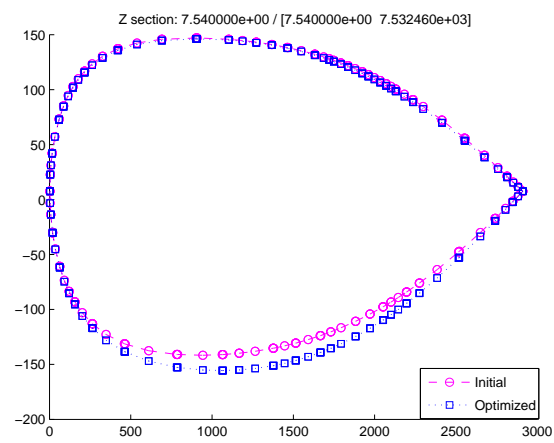

b) Mid-chord

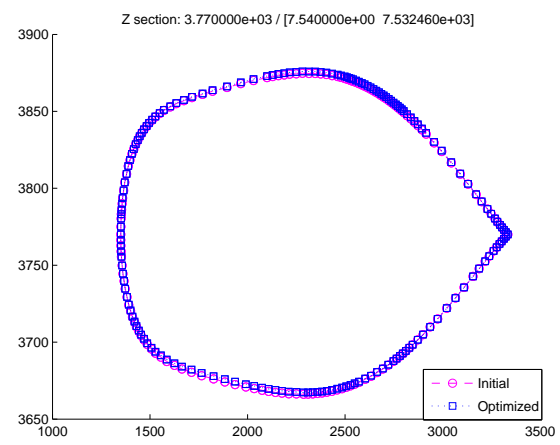

c) Wing tip

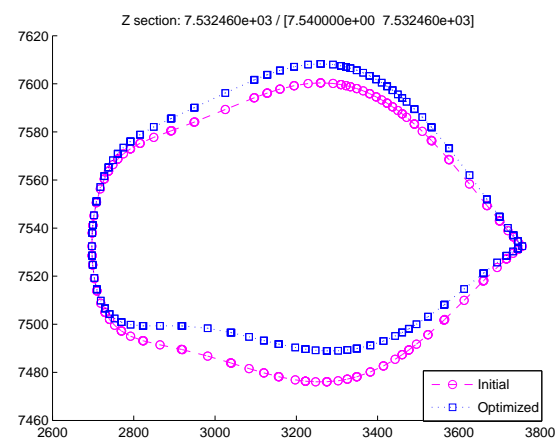

Figure 14. Aerostructural shape optimization of a business jet wing; split of variables according to the orthogonal decomposition; cross-section variations at a) root, b) mid-chord, and c) wing tip. 
a) Initial aerodynamic optimum solution

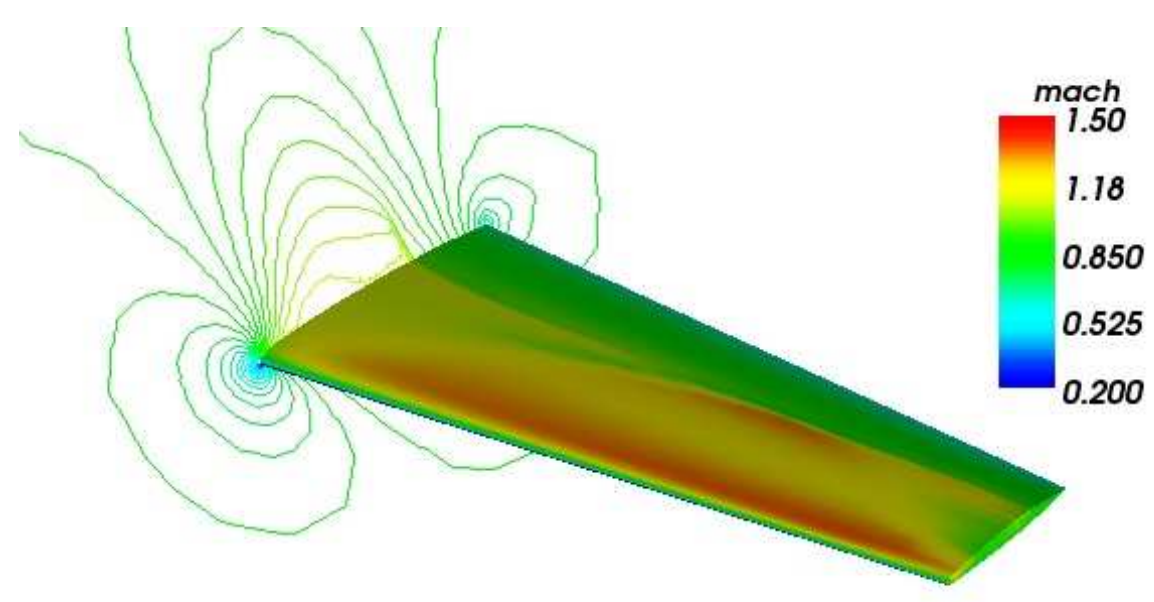

b) Aerostructural Nash game solution using the orthogonal decomposition

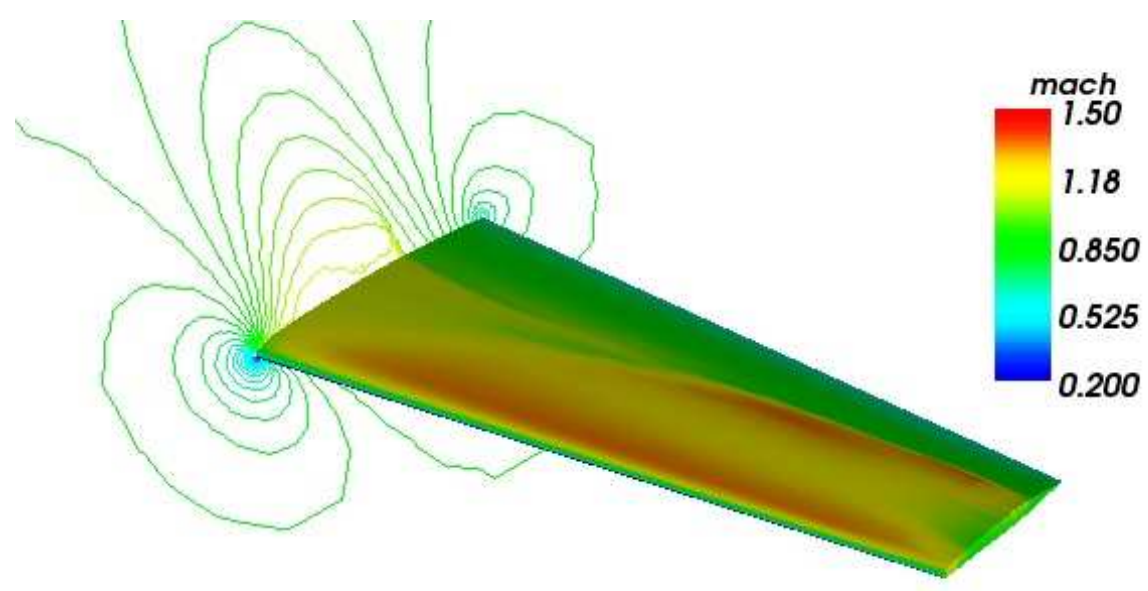

Figure 15. Geometrical configuration and Mach number field : a) initial aerodynamic optimum solution, and b) aerostructural Nash game solution using the orthogonal decomposition. 


\subsection{Conclusions}

The multiobjective optimization of an aerodynamic criterion concurrently with one or more criteria originating from other disciplines raises delicate problems to solve since the flowfields are very sensitive to parameter changes, such as perturbations in shape parameters, particularly when the flow is transonic or supersonic and contains shocks.

A theoretical formulation has been proposed for situations of this type, permitting to identify a suboptimal solution as a Nash-equilibrium solution between virtual players in charge of reducing independent criteria. An orthogonal decomposition of the design space is made to assign the player in charge of the secondary criterion a subspace of action, or territory, in the primary criterion has little sensitivity.

The method has been tested over a simplified testcase of aerostructural design of a business jet wing shape combining drag reduction under lift constraint in a transonic cruise configuration with the reduction of an integral of the stress over the structure. In this example, after a first phase of pure aerodynamic optimization, the primary criterion (drag) was modeled at convergence by an RBF neural network in order to approximate gradients and Hessians necessary to the construction of the orthogonal basis. This basis was then used as the support of a dynamic Nash game in a novel formulation.

The numerical experiments, taken from B. Abou El Majd's doctoral thesis, have clearly demonstrated the superiority of concurrent optimizations realized using the orthogonal decomposition as a support, in terms of asymptotic convergence stability, and achieved performance as well.

\section{Acknowledgements}

The author wishes to express his warmest thanks to his collaborators in the INRIA Opale Project-Team, and particularly to those whose results illustrate this chapter : B. Abou El Majd (aerostructural optimization), N. Blaszka-Marco (two-point aerodynamic optimization by the NSGA), Z. Tang (two-point aerodynamic optimization by a Nash game). Special thanks are also due to A. Habbal of the University of Nice - Sophia Antipolis and Opale, who originally proposed the formulation of the aerostructural concurrent problem, and actively participated in fruitful discussions on Nash games. 


\subsection{Bibliography}

[Abo 07] AвоU El MAJd B., Algorithmes hiérarchiques et stratégies de jeux pour l'optimisation multidisciplinaire - Application à l'optimisation de la voilure d'un avion d'affaires, PhD thesis, Université de Nice-Sophia Antipolis, 2007.

[Abo 08] Abou El Majd B., Désidéri J.-A., Duvigneau R., "Shape design in Aerodynamics: parameterization and sensitivity", vol. 17, Chapter Multilevel Strategies for Parametric Shape Optimization in Aerodynamics, Revue Européenne de Mécanique Numérique European Journal of Computational Mechanics, 2008.

[ATT 07] Attouch H., Redont P., Soubeyran A., "A New Class of Alternating Proximal Minimization Algorithms With Costs-To-Move”, SIAM J. Optim., vol. 18, num. 3, p. 10611081, 2007.

[BAS 95] Basar T., Olsder G. J., Dynamic Noncooperative Game Theory, Academic, Bodmin, Cornwall, Great Britain, 1995.

[CHA 07] Chandrashekarappa P., Duvigneau R., Radial Basis Functions and Kriging Metamodels for Aerodynamic Optimization, Research Report num. 6151, INRIA, 2007.

[DÉS 07a] DÉSIDÉRI J.-A., Split of Territories in Concurrent Optimization, Research Report num. 6108, INRIA, October 2007, URL: https://hal.inria.fr/inria-00127194 (version 6).

[DÉS 07b] DÉsidéri J.-A., Abou El Majd B., Janka A., "Nested and Self-Adaptive Bézier Parameterizations for Shape Optimization”, J. Comput. Phys., 2007, in press.

[DUV 07] Duvigneau R., "Meta-Modeling for Robust Design and Multi-Level Optimization", 42e Colloque d'Aérodynamique Appliquée, Couplages et Optimisation Multidisciplinaires, INRIA Sophia Antipolis, 19-21 March 2007, 2007.

[GOL 89] Goldberg D., Genetic Algorithms in Search, Optimization and Machine Learning, Addison Wesley Company Inc., 1989.

[MAR 99] MARCo N., DÉSIDÉRI J.-A., LANTERI S., Multi-Objective Optimization in CFD by Genetic Algorithms, Report num. 3686, INRIA, 1999.

[MAR 00] Marco N., Lanteri S., Désidéri J.-A., Mantel B., PÉriaux J., "A parallelized Genetic Algorithm for a 2-D shape optimum design problem", Surveys on Mathematics for Industry, vol. 9, p. 207-221, 2000.

[MAR 08] MARCELET M., Etude et mise en œuvre d'une méthode d'optimisation de forme couplant simulation numérique en aérodynamique et en calcul de structure, $\mathrm{PhD}$ thesis, Ecole Nationale d'Arts et Métiers, Paris, 2008.

[MIE 99] MietTinen K. M., Nonlinear Multiobjective Optimization, Kluwer Academic Publishers, Boston/London/Dordrecht, 1999.

[NAS 51] NASH J. F., "Non-Cooperative Games", Annals of Mathematics, vol. 54, num. 2, p. 286-295, 1951.

[NEL 65] Nelder J. A., Mead R., “A Simplex Method for Function Minimization”, Computer Journal, vol. 7, p. 308-313, 1965.

[SAM 00] SAMAREH J. A., "Multidisciplinary Aerodynamic-Structural Shape Optimization Using Deformation (MASSOUD)", 8th AIAA/NASA/USAF/ISS, MO Symposium 
36 Multidisciplinary Design Optimization in Computational Mechanics

on Multidisciplinary Analysis and Optimization, September 6-8, 2000/Long Beach, CA, num. 4911AIAA-2000, 2000.

[SRI 95] SRINIVAS N., Deb K., "Multi-objective function optimization using non-dominated sorting genetic algorithms", Evolutionary Computation, vol. 2, num. 3, Page221 248, 1995.

[TAN 07] TAng Z., Désidéri J.-A., Périaux J., "Multi-Criterion Aerodynamic ShapeDesign Optimization and Inverse Problems Using Control Theory and Nash Games", Journal of Optimization Theory and Applications, 2007.

[WAN 01] WANG J., Optimisation Distribuée Multicritère par Algorithmes Génétiques et Théorie des Jeux \& Application à la Simulation Numérique de Problèmes d'Hypersustentation en Aérodynamique, PhD thesis, University of Paris 6, 2001, Spéc.: Math. App. 